\title{
Whole blood profiling of leprosy type 1 (reversal) reactions highlights prominence of innate immune response genes
}

\author{
Jamile Leão Rêgo ${ }^{2}$, Nadja de Lima Santana ${ }^{2}$, Paulo Roberto Lima Machado 1,2, Marcelo Ribeiro-Alves ${ }^{3}$, \\ Thiago Gomes de Toledo-Pinto ${ }^{3}$, Léa Cristina Castellucci ${ }^{1,2,4^{*}}$ (D) and Milton Ozório Moraes ${ }^{3}$
}

\begin{abstract}
Background: The major factors contributing for nerve damage and permanent disabilities in leprosy are type 1 or reversal reactions (RR) and type 2 or erythema nodosum leprosum (ENL). Gene profiling of leprosy reactions have shown that different pathways are activated during the course of reactions, which is consistent with the exacerbated immune response exhibited by these patients.

Methods: We used qPCR to screen a panel of 90 genes related to the immune response in leprosy in RNA-derived peripheral leukocytes of patients with $(N=94)$ and without leprosy reactions $(N=57)$ in order to define expression signatures correlated to RR or ENL.

Results: Our results show that there is a marked signature for RR in the blood, comprising genes mostly related to the innate immune responses, including type I IFN components, autophagy, parkins and Toll like receptors. On the other hand, only Parkin was differentially expressed in the ENL group.

Conclusions: The data put together corroborates previous work that brings evidence that an acute uncontrolled exacerbated immune response designed to contain the spread of $\mathrm{M}$. leprae antigens might be cause of RR pathogenesis. Identifying a blood profile useful to predict leprosy reactions prior to its development might help to reduce the morbidity associated to this disabling disease.
\end{abstract}

Keywords: Leprosy reactions; gene expression; profile, Parkin, Pro-inflammatory, Type-I IFN, OASL

\section{Background}

Leprosy is a chronic infectious disease caused by Mycobacterium leprae. The bacilli invades Schwann cells and macrophages of the skin leading the tissue injury, which is the major reason for its pathogenesis [1-3]. Leprosy presents a wide variety of clinical presentations, including the indeterminate (I), tuberculoid (TT), borderline (BT, BB, BL) and lepromatous (LL) forms. In addition, about $20-50 \%$ of leprosy patients, depending on the population studied, can be affected by acute inflammatory episodes known as leprosy reactions, as so called type 1 (Reversal Reaction) or type 2 (Erythema Nodosum

\footnotetext{
* Correspondence: leacastel@hotmail.com

${ }^{1}$ Instituto Nacional de Ciência e Tecnologia em Doenças Tropicais, Salvador, Brazil

${ }^{2}$ Programa de Pós-graduação em Ciências da Saúde da Universidade Federal da Bahia, Salvador, Brazil

Full list of author information is available at the end of the article
}

Leprosum- ENL) $[4,5]$. Either RR or ENL are observed in all borderline forms prior, during or after completion of multidrug therapy. RR involves the active participation of $\mathrm{T}$ lymphocytes and abrupt episodes of intense local delayed-type hypersensitivity to $M$. leprae in skin and/or nerves. On the other hand, ENL is typical of the BL and LL forms and is correlated to a systemic reaction involving a cytokine storm and also deposition of immune complexes in skin and organs $[6,7]$. Regardless its type, leprosy reactions are an important contributing factor of nerve damage among patients with leprosy. The identification of host-derived biomarkers correlated to leprosy reactions might point out new tests to predict increased risk of developing the occurrence of reactional episodes thus helping to prevent its irreversible sequels. There are only a few transcriptomic studies searching for genes related to the development of leprosy reactions. Among these, a role 
for pro and anti-inflammatory regulators, IFN-induced genes, complement components, among others have been described $[5,6,8]$.

\section{Methods}

In this study we analyzed the expression of a panel of relevant immune response genes in RNA-derived from peripheral blood leukocytes of leprosy patients with and without reactions in order to identify gene expression signatures associated with either RR or ENL. One hundred and fifty one cDNA samples of patients divided into three groups were used: 57 patients with no evidence of reactions (hereinafter referred to as No Reaction Group - NR), 50 patients with RR and 44 patients with ENL. Subjects were diagnosed according to the Brazilian's Ministry of Health guidelines in the leprosy outpatient clinics from Hospital Universitário Professor Edgard Santos and Hospital Couto Maia in the city of Salvador-Bahia, Brazil. Patients were classified according to a Ridley-Jopling classification and by the WHO field classification $[9,10]$, as previously reported for studies of patients recruited from this hospital in Salvador [11]. Detailed complementary data about the participants are described in Table 1. Written informed consent was obtained from all patients after approval of the study by the Ethics Committee from the Federal University of Bahia (number 891.963). Peripheral leukocytes from patients free of immunosuppressants such as thalidomide or prednisone were homogenized in TRIzol (Thermo Fisher Scientific). RNA was extracted using the PureLink ${ }^{\mathrm{Tm}}$ RNA Mini Kit (Thermo Fisher Scientific) and the total RNA concentration was determined in optical density spectrophotometer (260 and $280 \mathrm{~nm}$ ). The cDNA conversion was performed using the High Capacity cDNA Reversion Transcription Kit (Applied Biosystems) following the manufacturer's instructions. The expression of 90 target genes and 4 normalizing genes was performed by medium- throughput quantitative q-PCR using the microfluidic system Biomark (Fluidigm, CA). The analysis was performed from the real-time fluorescence accumulation data of each sample $(\Delta \mathrm{Rn})$, using the logistic function adjustment of four parameters to represent each amplification curve by the library of qpcR ( $R$ Development Core Team, 2009) version 2.922. Results: After filtering by QC, 35 genes were excluded and 55 analyzed. We first compared the paucibacillary $(\mathrm{PB})$ versus multibacillary (MB) leprosy within the unreactional (NR) group checking for differences regarding these two disease poles. This analysis did not show any significant differences $(p<0.05$, data not shown). Nevertheless, there was a differential pattern of gene expression between the NR and RR group as shown in Table 2. A set of genes belonging to different pathways that includes the parkin pathway, pattern recognition receptors (PRRs), type I IFNs precursors, inflammatory cytokines and chemokines and
Table 1 Demographic and clinical characteristics of leprosy patients

\begin{tabular}{llll}
\hline A - Characteristics of the sample & & \\
& N individuals & & Age, years \\
& \pm SD & Gender M:F \\
Cases / Reaction & 94 & $44.05(13.31)$ & $58: 36$ \\
Controls / No Reaction & 57 & $45.21(14.98)$ & $28: 29$
\end{tabular}

\begin{tabular}{lll} 
B - Clinical characteristics of the cohort $^{\mathrm{a}}$ & & \\
Clinical phenotype & $n$ & $(\%)$ \\
Tuberculoid (TT) & 20 & $(13)$ \\
Borderline tuberculoid (BT) & 30 & $(20)$ \\
Borderline (BB) & 18 & $(12)$ \\
Borderline lepromatous (BL) & 17 & $(11)$ \\
Lepromatous (LL) & 49 & $(32)$ \\
Indeterminate leprosy (I) & 11 & $(7)$ \\
Other forms (Neural) & 6 & $(4)$ \\
Total\# & 151 \\
C - Patients with reaction episode & \\
RR & & \\
ENL & 50 \\
Total\# & 44 \\
D - Patients without reaction episode & \\
PB & 94 \\
MB & & \\
Total\# & & \\
\hline
\end{tabular}

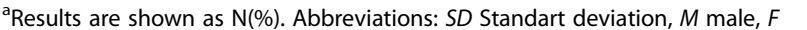
female, $P B$ paucibacillary, $M B$ multibacillary, $R R$ reversal reaction, $E N L$ erythema nodosum leprosum. Patients were also classified under leprosy clinical spectrum according to Ridley \& Jopling [9]

eicosanoid metabolism were significantly more expressed in RR patients as compared to the NR group (Fig. 1). This peculiar inflammatory signature for type 1 reaction has been described in previous works $[5,8]$ that also underpinned a mixed immune activation that seems to lead to the RR pathogenesis. On the other hand, only PARK2 was significantly more expressed in leucocytes of ENL compared to unreactional patients $(\log \mathrm{FC}=2.13$ e $p=0.04)$, as well as TLR7 between RR and ENL subjects $(\operatorname{logFC}=-$ 2.72 e $p=0.02$ ).

\section{Discussion}

Our RR signature corroborates data showing that $M$. leprae components and host cell destruction continue to stimulate the immune response in a sudden and acute manner during RR. Most pathogen-associated molecular patterns (PAMPs) and damage-associated molecular pattern molecules (DAMPs) bind specific PRRs such as Toll-like receptors and NOD-like receptors to orchestrate both, autophagy and IFN signaling $[12,13]$. We hypothesized that the 
Table 2 Normalized gene expression values of whole blood leukocytes samples of leprosy patients with reactions $(n=94)$ and leprosy patients without reactions $(n=57)$

\begin{tabular}{|c|c|c|c|}
\hline \multicolumn{4}{|c|}{ Reaction vs No Reaction } \\
\hline Gene & Description & log fold change & p.value* \\
\hline CCL2 & C-C Motif Chemokine Ligand 2 & 4.04 & 0.0016 \\
\hline PARK & parkinson protein 2, E3 ubiquitin protein ligase & 3.27 & 0.0036 \\
\hline ALOX5 & Arachidonate 5-Lipoxygenase & 2.99 & 0.0108 \\
\hline TLR7 & Toll Like Receptor 7 & 2.98 & 0.011 \\
\hline LRRK2 & Leucine Rich Repeat Kinase 2 & 3.09 & 0.0161 \\
\hline IFNB & interferon beta 1 & 2.36 & 0.0228 \\
\hline TLR10 & Toll Like Receptor 10 & 3.07 & 0.0236 \\
\hline IL18 & Interleukin 18 & 2.34 & 0.0326 \\
\hline TLR3 & Toll Like Receptor 3 & 2.74 & 0.0338 \\
\hline CLEC5A & C-Type Lectin Domain Containing 5A & 2.81 & 0.0446 \\
\hline \multicolumn{4}{|c|}{ RR vs No Reaction } \\
\hline CCL2 & C-C Motif Chemokine Ligand 2 & 4.31 & 0.0002 \\
\hline TLR7 & Toll Like Receptor 7 & 3.81 & 0.0012 \\
\hline PARK & parkinson protein 2, E3 ubiquitin protein ligase & 3.16 & 0.002 \\
\hline ALOX5 & Arachidonate 5-Lipoxygenase & 3.32 & 0.0044 \\
\hline LRRK2 & Leucine Rich Repeat Kinase 2 & 3.28 & 0.0056 \\
\hline IFNB & interferon beta 1 & 2.64 & 0.0104 \\
\hline TLR10 & Toll Like Receptor 10 & 3.08 & 0.0122 \\
\hline TLR3 & Toll Like Receptor 3 & 3.04 & 0.0122 \\
\hline IL18 & Interleukin 18 & 2.65 & 0.014 \\
\hline OAS1 & 2'-5'-oligoadenylate synthetase 1 & 2.51 & 0.0376 \\
\hline IL15 & Interleukin 15 & 2.42 & 0.0446 \\
\hline \multicolumn{4}{|c|}{ ENL vs No Reaction } \\
\hline PARK & parkinson protein 2, E3 ubiquitin protein ligase & 2.13 & 0.0436 \\
\hline \multicolumn{4}{|l|}{ ENL vs RR } \\
\hline TLR7 & Toll Like Receptor 7 & -2.72 & 0.0214 \\
\hline
\end{tabular}

${ }^{*}$ The genes were defined as differentially expressed by the criterion of $p$-value adjusted for multiple comparisons. Bayesian statistical analysis used a log fold change cutoff of $>1$ and adjusted $p$ value of $<0.05$. - Abbreviations: $R R$ reversal reaction, ENL erythema nodosum leprosum

continued binding of PAMPs and DAMPs to TLRs caused by the pathogen components after killing destruction provides the necessary trigger for maintenance of the inflammatory process. The stimulation of innate mechanisms that comprise genes with autophagic activities such as PARK and $L R R K 2$, in addition to the type I IFNs in the beginning of the process seems to be activated in order to clear killed mycobacteria, but it is unbalanced and exacerbated. Regarding the IFNs, the genes IFNB and OAS1 (2-5 'oligoadenylate synthetase-1 gene) had a greater expression in RR samples. $O A S L$ was also shown to be upregulated in $M$. leprae-infected human macrophage cell lineages, primary monocytes, and skin lesion from patients with a disseminated form of leprosy; whereas $O A S L$ knock down was associated with decreased viability of $M$. leprae and upregulation of autophagy levels [14]. Additionally, the chemokine CCL2 was the most expressed gene in our RR group. Recent reports have linked the STING signaling, type I IFN and CCL2 activation [14, 15]. During mycobacterial infection, this chemokine can be produced in a STING-dependent manner and it is actively involved in the recruitment of monocytes to the infection site [15] and also related to mycobacterial survival within macrophages [14]. Other works have pointed the participation of CCL2 in the pathogenesis of several inflammatory disorders such as atherosclerosis and autoimmune diseases [16-18]. Here, we could speculate that a prominent triggering of STING signaling and high expression of type I IFN and CCL2 may contribute to the attraction of immune cells and enhancement of inflammatory response during leprosy reaction.

Type 1 reaction or RR is caused by an amplified immune response possibly triggered by fragmented bacillary antigens available in the cell medium [19]. The main issue however, is that a dysregulated process of gene activation, aiming to 


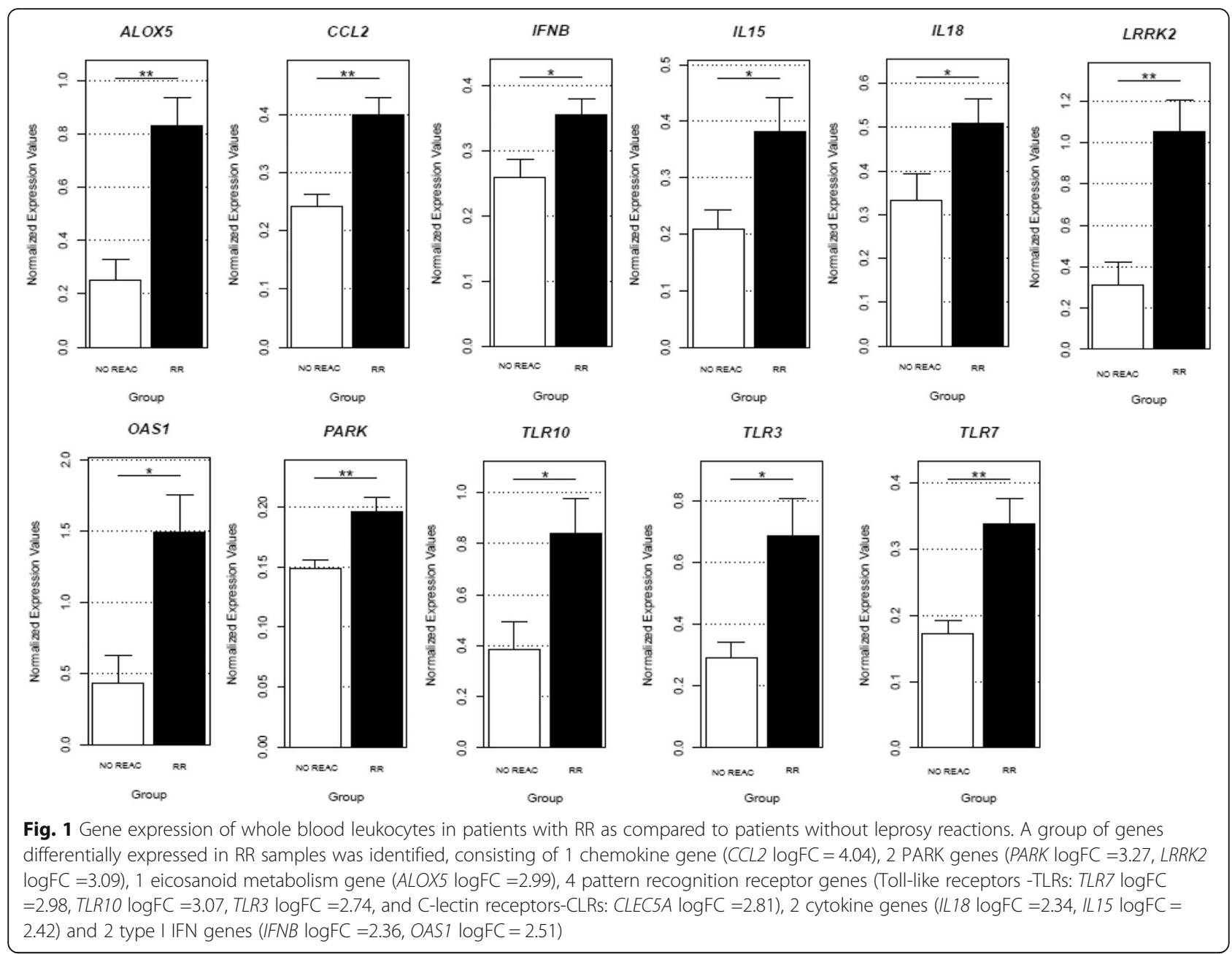

contain the progress of $M$. leprae and eliminate the infection, will lead to the nerve and tissue damage. Indeed, persons with history of RR can keep an altered response to $M$. leprae antigens that differs from patients with unreactional leprosy for years after resolution of RR [8]. Additionally, our results show that the expression of TLR3, TLR7 and $T L R 10$ were significantly increased in the reactions per se as well as in RR with TLR7 and TLR10 corroborating with data that fragments of bacterial destruction may be giving continuity to the characteristic inflammatory process of both reactional episodes in leprosy. On the other hand, ENL is characterized by a systemic inflammatory reaction. In this case, it might be possible that other set of genes related to the humoral immune response would be more active in these leucocytes. We need to expand our panel in order to identify which profile explains ENL.

\section{Conclusion}

Overall our data strength previous data and reinforces a signature for RR that could help to guide future studies for developing tools to predict this condition among leprosy patients. Personalizing the treatment of individuals susceptible to the development of reactions will help increase the effectiveness of treatment and reduce morbidity and disability in leprosy.

\section{Acknowledgements}

We thank the staff of outpatients leprosy clinics from Hospital Universitário Professor Edgard Santos and Hospital Couto Maia for the support in the subjects recruitment and sample collection.

\section{Funding}

National Institute of Science and Technology in Tropical Diseases, Brazil $\left(\mathrm{N}^{\circ}\right.$ 573839/2008-5), CNPq, Brazil (Nº4277/2012-8 and Nº 309397/2013-8) and FAPERJ ( $N^{\circ}$ E9/2016). We also thank CAPES by granting scholarships.

\section{Availability of data and materials}

The datasets used and analyzed during the current study are available from the corresponding author on reasonable request.

\section{Authors' contributions}

PM diagnosed, treated and included the patients in the study. JLR and NLS collected the samples and extracted the RNA from the patients. JLR and TGT performed the gene expression experiments. MRA and TGT analyzed the data. LCC supervised the samples processing and wrote the manuscript. MOM supervised the data and manuscript preparation. All authors read and approved the final manuscript. 


\section{Ethics approval and consent to participate}

The study has been approved by the Ethical Committee of the Hospital Universitário Prof. Edgar Santos, Federal University of Bahia, number 891.963. All patients read and signed an informed consent form.

\section{Consent for publication}

Not applicable.

\section{Competing interests}

The authors declare that they have no competing interests.

\section{Publisher's Note}

Springer Nature remains neutral with regard to jurisdictional claims in published maps and institutional affiliations.

\section{Author details}

${ }^{1}$ Instituto Nacional de Ciência e Tecnologia em Doenças Tropicais, Salvador, Brazil. ${ }^{2}$ Programa de Pós-graduação em Ciências da Saúde da Universidade Federal da Bahia, Salvador, Brazil. ${ }^{3}$ Fundação Oswaldo Cruz-FIOCRUZ, Rio de Janeiro, Brazil. ${ }^{4}$ Hospital Universitário Professor Edgard Santos, Serviço de Imunologia, Rua João das Botas, S/N, $5^{\circ}$ andar, Salvador, Bahia 40.110-160, Brazil.

Received: 5 February 2018 Accepted: 20 August 2018

Published online: 24 August 2018

\section{References}

1. Agrawal A, et al. Neurological manifestations of Hansen's disease and their management. Clin Neurol Neurosurg. 2005;107(6):445-54.

2. Eichelmann K., G.G.S., Salas-Alanis JC, Ocampo-Candiani J., Leprosy An update: definition, pathogenesis, classification, diagnosis, and treatment. Actas Dermo-Sifiliográficas, 2013. 104(7): p. 554-563.

3. Lastória JC, de Abreu MAMM. Leprosy: review of the epidemiological, clinical, and etiopathogenic aspects - part 1. An Bras Dermatol. 2014;89(2):205-18.

4. Rêgo JL, et al. The role of ERBB2 gene polymorphisms in leprosy susceptibility. Braz J Infect Dis. 2015;19:206-8.

5. Geluk A, et al. Longitudinal immune responses and gene expression profiles in type 1 leprosy reactions. J Clin Immunol. 2014;34(2):245-55.

6. Dupnik KM, et al. Transcriptional changes that characterize the immune reactions of leprosy. J Infect Dis. 2015;211(10):1658-76.

7. Moraes MO, et al. Anti-inflammatory drugs block cytokine mRNA accumulation in the skin and improve the clinical condition of reactional leprosy patients. J Investig Dermatol. 2000;115(6):935-41.

8. Orlova $\mathrm{M}$, et al. Gene set signature of reversal reaction type I in leprosy patients. PLoS Genet. 2013;9(7):e1003624.

9. Ridley D, Jopling W. Classification of leprosy according to immunity. A fivegroup system. Int I Lepr Other Mycobact Dis. 1966;34(3):255-73.

10. WHO, Action Programme for the Elimination of Leprosy. Efficacy of single dose multi drug therapy for the treatment of single-lesion paucibacillary leprosy. Geneva : World Health Organization, 1997. 69(2): p. 121-129.

11. Machado PRL, et al. Viral co-infection and leprosy outcomes: a cohort study. PLoS Negl Trop Dis. 2015;9(8):e0003865.

12. Zhang F, et al. Genomewide association study of leprosy. N Engl J Med. 2009;361(27):2609-18.

13. Saxena M, Yeretssian G. NOD-like receptors: master regulators of inflammation and cancer. Front Immunol. 2014:5:327.

14. de Toledo-Pinto TG, et al. STING-dependent 2'-5' Oligoadenylate Synthetase-like production is required for intracellular Mycobacterium leprae survival. J Infect Dis. 2016;214(2):311-20.

15. Cambier $\mathrm{CJ}$, et al. Phenolic glycolipid facilitates mycobacterial escape from Microbicidal tissue-resident macrophages. Immunity. 2017;47(3):552-565.e4.

16. Tracy OC, Lubor B, Mathias H. CCL2-CCR2 signaling in disease pathogenesis. Endocrine, Metabolic \& Immune Disorders - Drug Targets. 2015;15(2):105-18.

17. Gosling J, et al. MCP-1 deficiency reduces susceptibility to atherosclerosis in mice that overexpress human apolipoprotein B. J Clin Invest. 1999;103(6): 773-8.

18. Behfar S, et al. A brief look at the role of monocyte chemoattractant protein-1 (CCL2) in the pathophysiology of psoriasis. Cytokine. 2017

19. Mendonça VA, et al. Immunology of leprosy. An Bras Dermatol. 2008;83(4): $343-50$

Ready to submit your research? Choose BMC and benefit from:

- fast, convenient online submission

- thorough peer review by experienced researchers in your field

- rapid publication on acceptance

- support for research data, including large and complex data types

- gold Open Access which fosters wider collaboration and increased citations

- maximum visibility for your research: over $100 \mathrm{M}$ website views per year

At BMC, research is always in progress.

Learn more biomedcentral.com/submissions 